Kragujevac Journal of Mathematics

Volume 45(2) (2021), Pages 309-322.

\title{
SUMMATION-INTEGRAL TYPE OPERATORS BASED ON LUPAŞ-JAIN FUNCTIONS
}

\author{
NESIBE MANAV ${ }^{1}$ AND NURHAYAT ISPIR ${ }^{1}$
}

\begin{abstract}
We introduce a genuine summation-integral type operators based on Lupaş-Jain type base functions related to the unbounded sequences. We investigated their degree of approximation in terms of modulus of continuity and $\mathcal{K}$-functional for the functions from bounded and continuous functions space. Furthermore, we give some theorems for the local approximation properties of functions belonging to Lipschitz class. Also, we give Voronovskaja theorem for these operators.
\end{abstract}

\section{INTRODUCTION}

Inspiring by Lupaş's paper [12], Agratini studied the following operators

$$
L_{n}(f, x)=2^{-n x} \sum_{k=0}^{\infty} \frac{(n x)_{k}}{2^{k} k !} f\left(\frac{k}{n}\right)
$$

where $f \in C[0, \infty), C[0, \infty)$ is the space of all real valued continuous functions on $[0, \infty)$ in [1]. Agratini gave some estimations for rates of convergence, an asymptotic formula and a reobtained version by using probabilistic methods at the same study. Also, he introduced a Kantorovich and a Durrmeyer modifications of the operators (1.1). Agratini, in [2], gave some estimations on the Kantorovich variant of the operators (1.1) by using modulus of smoothness. Moreover, he investigated rate of convergence by the step weight function of Lupaş operators, for local Lipschitz class functions. Also, he gave some approximation properties of the operators given by (1.1) using probabilistic methods.

Key words and phrases. Lupaş-Jain functions, summation-integral type operators, moduli of continuity, $\mathcal{K}$-functional, Voronovskaja theorem.

2010 Mathematics Subject Classification. Primary: 41A10. Secondary: 41A25, 41A36.

DOI 10.46793/KgJMat2102.309M

Received: August 22, 2018.

Accepted: December 17, 2018. 
In [3], Erençin and Taşdelen introduced a generalization of the operators (1.1) with the help of increasing and unbounded sequences of positive numbers $\left(a_{n}\right),\left(b_{n}\right)$. They studied weighted approximation properties of these generalized operators. Later, in [4], they studied convergence properties of the Kantorovich type version of these operators. By using the modulus of continuity and Peetre's $\mathcal{K}$-functional, they gave the rate of convergence of these operators. Also, they investigated convergence properties for the functions from local Lipschitz class.

In [9], we generalized the operators (1.1) based on Lupaş base function by using the sequences $\left(a_{n}\right),\left(b_{n}\right)$ as follows

$$
L_{a_{n}, b_{n}}(f ; x)=2^{-\frac{a_{n}}{b_{n}} x} \sum_{k=0}^{\infty} \frac{\left(\frac{a_{n}}{b_{n}} x\right)_{k}}{2^{k} k !} f\left(\frac{b_{n}}{a_{n}} k\right),
$$

where $\left(a_{n}\right),\left(b_{n}\right)$ are unbounded and increasing sequences of positive real numbers such that

$$
\lim _{n \rightarrow \infty} \frac{b_{n}}{a_{n}}=0 \quad \text { and } \quad \frac{b_{n}}{a_{n}} \leq 1 .
$$

We gave and investigated some basic results for these operators. Also, using Lupaş and Szász basis functions we defined summation-integral type operators

$$
D_{a_{n}, b_{n}}(f ; x)=\frac{a_{n}}{b_{n}} \sum_{k=0}^{\infty} l_{n, k}(x) \int_{0}^{\infty} P_{n, k}(u) f(u) d u,
$$

where $P_{n, k}(x)=e^{-\frac{a_{n}}{b_{n}} x} \frac{\left(\frac{a_{n}}{b_{n}} x\right)^{k}}{k !}, l_{n, k}(x)=\frac{\left(\frac{a_{n}}{b_{n}} x\right)_{k}}{2^{k} k !} 2^{-\frac{a_{n}}{b_{n}} x}$. Then, we gave the degree of approximation of these operators in terms of Ditzian-Totik modulus of smoothness and corresponding $\mathcal{K}$-functional. Also, we examined the convergence by using the Lipschitz class functions and we gave some results in weighted spaces.

Govil et al. in [5], introduced a modification of Lupaş operators with weight of Szász basis functions. They investigated the rate of convergence for the functions which have bounded derivatives. In addition, they gave a new modification of the Lupaş operators as follows

$$
D_{n}(f ; x)=n \sum_{k=1}^{\infty} l_{n, k}(x) \int_{0}^{\infty} p_{n, k-1}(u) f(u) d u+l_{n, 0}(x) f(0), \quad x \geq 0,
$$

where

and

$$
p_{n, k-1}(x)=e^{-n t} \frac{(n x)^{k-1}}{(k-1) !}
$$

$$
l_{n, k}(x)=\frac{(n x)_{k}}{2^{k} k !} 2^{-n x}
$$


Jain in [10], introduced the modified form of the Szász-Mirakjan operator as follows

$$
P_{n}^{\beta}(f ; x)=\sum_{k=0}^{\infty} p_{n, k}^{\beta}(x) f\left(\frac{k}{n}\right), \quad x \geq 0,
$$

where the operators based on certain parameter $0 \leq \beta<1$ and the base function

$$
p_{n, k}^{\beta}(x)=e^{-n x+k \beta} \frac{n x(n x+k \beta)^{k-1}}{k !} .
$$

The rate of approximation of operators given by (1.4), for some values of $n$, is better than the rate of approximation of operators Szász-Mirakjan.

Gupta and Greubel established the Durrmeyer variant of the operators (1.4) as follow

$$
D_{n}^{\beta}(f ; x)=\sum_{k=1}^{\infty}\left(\int_{0}^{\infty} p_{n, k-1}^{\beta}(u) d u\right)^{-1} p_{n, k}^{\beta}(x) \int_{0}^{\infty} p_{n, k-1}^{\beta}(u) f(u) d u+e^{-n x} f(0)
$$

and investigated some approximation properties in [6].

Inspiring by the previous studies, we define a genuine summation-integral type operators by using Jain and Lupaş base functions for integrable functions as follows

$$
\begin{aligned}
& D_{a_{n}, b_{n}}^{[\beta]}(f ; x) \\
= & \sum_{k=1}^{\infty}\left(\int_{0}^{\infty} \theta_{\beta}\left(k-1, \frac{a_{n}}{b_{n}} u\right) d u\right)^{-1} l_{n, k}^{*}(x) \int_{0}^{\infty} \theta_{\beta}\left(k-1, \frac{a_{n}}{b_{n}} u\right) f(u) d u+2^{-\frac{a_{n}}{b_{n}} x} f(0),
\end{aligned}
$$

where $f \in C[0, \infty)$ is integrable function, $l_{n, k}^{*}(x)=\frac{\left(\frac{a_{n}}{b_{n}}\right)_{k}}{2^{k} k !} 2^{-\frac{a_{n}}{b_{n}} x},\left(a_{n}\right)$ and $\left(b_{n}\right)$ are unbounded and increasing sequences of positive real numbers satisfying the condition (1.3) and the Jain-type base function is

$$
\theta_{\beta}\left(k, \frac{a_{n}}{b_{n}} x\right)=\frac{a_{n}}{b_{n}} x\left(\frac{a_{n}}{b_{n}} x+k \beta\right)^{k-1} \frac{e^{-\left(\frac{a_{n}}{b_{n}} x+k \beta\right)}}{k !},
$$

for $x \in[0, \infty), n \in \mathbb{N}$ and $\beta \in[0,1)$. Here, by considering the definition of $\theta_{\beta}$ we see that $\sum_{k=0}^{\infty} \theta_{\beta}\left(k, \frac{a_{n}}{b_{n}} x\right)=1$.

In addition, if we take $\langle f, g\rangle=\int_{0}^{\infty} f(t) g(t) d t$ at the definition of the operators $D_{a_{n}, b_{n}}^{[\beta]}$, we can write these operators as follow (see $[6,7]$ )

$$
D_{a_{n}, b_{n}}^{[\beta]}(f ; x)=\sum_{k=1}^{\infty} \frac{\left\langle\theta_{\beta}\left(k-1, \frac{a_{n}}{b_{n}} u\right), f(t)\right\rangle}{\left\langle\theta_{\beta}\left(k-1, \frac{a_{n}}{b_{n}} u\right), 1\right\rangle} l_{n, k}^{*}(x)+2^{-\frac{a_{n}}{b_{n}} x} f(0) .
$$

In this paper, we give the degree of approximation of these operators using the modulus of continuity and Peetre's $\mathcal{K}$-functional. Also, we give some theorems about local approximation and Voronovskaja theorem. 
1.1. Direct results for $D_{a_{n}, b_{n}}^{[\beta]}$ operators. In this section, we give some basic properties of the operators $D_{a_{n}, b_{n}}^{[\beta]}$ operators.

Lemma 1.1. For $0 \leq \beta<1$ we define

$$
P_{r}^{*}(k-1, \beta)=\frac{\left\langle\theta_{\beta}\left(k-1, \frac{a_{n}}{b_{n}} u\right), t^{r}\right\rangle}{\left\langle\theta_{\beta}\left(k-1, \frac{a_{n}}{b_{n}} u\right), 1\right\rangle},
$$

and we get the following

$$
\begin{aligned}
& P_{0}^{*}(k-1, \beta)=1, \\
& P_{1}^{*}(k-1, \beta)=\frac{b_{n}}{a_{n}}\left[(1-\beta) k+\frac{\beta(2-\beta)}{1-\beta}\right], \\
& P_{2}^{*}(k-1, \beta)=\left(\frac{b_{n}}{a_{n}}\right)^{2}\left[(1-\beta)^{2} k^{2}+\left(1+4 \beta-2 \beta^{2}\right) k+\frac{\beta^{2}(3-\beta)}{1-\beta}\right] .
\end{aligned}
$$

The proof is obtained by method of Lemma 2 at [6].

Lemma 1.2. ([9, Lemma 1$])$. For $f \in C[0, \infty)$ and $x \in[0, \infty)$, the operators $L_{a_{n}, b_{n}}$ given by (1.2) satisfy the following conditions

$$
\begin{aligned}
& L_{a_{n}, b_{n}}\left(e_{0} ; x\right)=1, \\
& L_{a_{n}, b_{n}}\left(e_{1} ; x\right)=x, \\
& L_{a_{n}, b_{n}}\left(e_{2} ; x\right)=x^{2}+2 \frac{b_{n}}{a_{n}} x,
\end{aligned}
$$

where $e_{i}(x)=x^{i}, i=0,1,2$, and $\left(a_{n}\right),\left(b_{n}\right)$ are sequences of positive real numbers satisfying the condition (1.3).

Now, we give the following equalities for the test functions of the operators defined with (1.6).

Lemma 1.3. Let $e_{k}(x)=x^{k}, k=0,1,2$, and $\left(a_{n}\right),\left(b_{n}\right)$ are unbounded and increasing sequences of positive real numbers satisfying the condition (1.3). For each $x \in[0, \infty)$, the operators $D_{a_{n}, b_{n}}^{[\beta]}$ satisfy the following equalities

$$
\begin{aligned}
D_{a_{n}, b_{n}}^{[\beta]}\left(e_{0} ; x\right)=1 & \\
D_{a_{n}, b_{n}}^{[\beta]}\left(e_{1} ; x\right)= & x(1-\beta)+\frac{b_{n}}{a_{n}} \frac{\beta(2-\beta)}{(1-\beta)}, \\
D_{a_{n}, b_{n}}^{[\beta]}\left(e_{2} ; x\right)= & (1-\beta)^{2} x^{2}+3 \frac{b_{n}}{a_{n}} x+\left(\frac{b_{n}}{a_{n}}\right)^{2} \frac{\beta^{2}(3-\beta)}{(1-\beta)}, \\
D_{a_{n}, b_{n}}^{[\beta]}\left(e_{3} ; x\right)= & (1-\beta)^{3} x^{3}+3 \frac{b_{n}}{a_{n}}\left(9-15 \beta+9 \beta^{2}-3 \beta^{3}\right) x^{2} \\
& +\left(\frac{b_{n}}{a_{n}}\right)^{2}\left(6+14 \beta+16 \beta^{2}-2 \beta^{3}+\frac{3 \beta^{4}}{1-\beta}\right) x+\left(\frac{b_{n}}{a_{n}}\right)^{3} \frac{\beta^{2}(3-\beta)}{1-\beta},
\end{aligned}
$$




$$
\begin{aligned}
D_{a_{n}, b_{n}}^{[\beta]}\left(e_{4} ; x\right)= & (1-\beta)^{4} x^{4}+\frac{b_{n}}{a_{n}}\left(18-28 \beta+22 \beta^{2}-8 \beta^{3}+2 \beta^{4}\right) x^{3} \\
& +\left(\frac{b_{n}}{a_{n}}\right)^{2}\left(47-40 \beta+30 \beta^{2}-20 \beta^{3}+5 \beta^{4}\right) x^{2} \\
& +\left(\frac{b_{n}}{a_{n}}\right)^{3}\left(42-+30 \beta^{2}-20 \beta^{3}+20 \beta^{4}-\frac{10 \beta^{5}}{1-\beta}\right) x+\left(\frac{b_{n}}{a_{n}}\right)^{4} \frac{\beta^{4}(5-\beta)}{1-\beta} .
\end{aligned}
$$

Proof. Considering the definition of the operator (1.6), the properties of Pochammer symbol, and using the equality

$$
2^{\frac{a_{n}}{b_{n}} x}=\sum_{k=0}^{\infty} \frac{\left(\frac{a_{n}}{b_{n}} x\right)_{k}}{2^{k} k !}, \quad x \in[0, \infty)
$$

and by considering Lemma 1.1 and Lemma 1.2, we get

$$
D_{a_{n}, b_{n}}^{[\beta]}\left(e_{0} ; x\right)=\sum_{k=1}^{\infty} P_{0}^{*}(k-1, \beta) l_{n, k}^{*}(x)+2^{-\frac{a_{n}}{b_{n}} x} e_{0}(0)=\sum_{k=1}^{\infty} l_{n, k}^{*}(x)=L_{a_{n}, b_{n}}\left(e_{0} ; x\right)=1 .
$$

For $e_{1}$ and $e_{2}$ we have following results,

$$
\begin{aligned}
D_{a_{n}, b_{n}}^{[\beta]}\left(e_{1} ; x\right)= & \sum_{k=1}^{\infty} P_{1}^{*}(k-1, \beta) l_{n, k}^{*}(x)+2^{-\frac{a_{n}}{b_{n}} x} e_{1}(0) \\
= & \sum_{k=1}^{\infty} l_{n, k}^{*}(x)\left[\frac{b_{n}}{a_{n}}\left((1-\beta) k+\frac{\beta}{1-\beta}+1\right)\right] \\
= & \frac{b_{n}}{a_{n}}(1-\beta) \sum_{k=1}^{\infty} l_{n, k}^{*}(x)(k)+\frac{b_{n}}{a_{n}} \frac{\beta(2-\beta)}{1-\beta} \\
= & (1-\beta) L_{a_{n}, b_{n}}\left(e_{1} ; x\right)+\frac{b_{n}}{a_{n}} \frac{\beta(2-\beta)}{1-\beta} L_{a_{n}, b_{n}}\left(e_{0} ; x\right) \\
= & (1-\beta) x+\frac{b_{n}}{a_{n}} \frac{\beta(2-\beta)}{1-\beta}, \\
D_{a_{n}, b_{n}}^{[\beta]}\left(e_{2} ; x\right)= & \sum_{k=1}^{\infty} P_{2}^{*}(k-1, \beta) l_{n, k}^{*}(x)+2^{-\frac{a_{n}}{b_{n}} x} e_{2}(0) \\
= & \left(\frac{b_{n}}{a_{n}}\right)^{2}(1-\beta)^{2} \sum_{k=1}^{\infty} l_{n, k}^{*}(x) k^{2}+\left(\frac{b_{n}}{a_{n}}\right)^{2}\left(1+4 \beta-2 \beta^{2}\right) \sum_{k=1}^{\infty} l_{n, k}^{*}(x) k \\
& +\left(\frac{b_{n}}{a_{n}}\right)^{2} \frac{\beta^{2}(3-\beta)}{1-\beta} \sum_{k=1}^{\infty} l_{n, k}^{*}(x) \\
= & (1-\beta)^{2} L_{a_{n}, b_{n}}\left(e_{2} ; x\right)+\frac{b_{n}}{a_{n}}\left((1-\beta)^{2}+\left(2+4 \beta-2 \beta^{2}\right)\right) L_{a_{n}, b_{n}}\left(e_{1} ; x\right) \\
& +\left(\frac{b_{n}}{a_{n}}\right)^{2} \frac{\beta^{2}(3-\beta)}{(1-\beta)} L_{a_{n}, b_{n}}\left(e_{0} ; x\right)
\end{aligned}
$$




$$
=(1-\beta)^{2} x^{2}+3 \frac{b_{n}}{a_{n}} x+\left(\frac{b_{n}}{a_{n}}\right)^{2} \frac{\beta^{2}(3-\beta)}{(1-\beta)} .
$$

By using same method, if we keep to continue the calculations we can get the values of other test functions.

As a result of this lemma, we can give the central moments.

Lemma 1.4. For each $x \in[0, \infty)$ and the operators $D_{a_{n}, b_{n}}^{[\beta]}$, we have

$$
\begin{aligned}
& \mu_{n, 1}(x):=D_{a_{n}, b_{n}}^{[\beta]}((t-x) ; x)=x(-\beta)+\frac{b_{n}}{a_{n}} \frac{\beta(2-\beta)}{(1-\beta)}, \\
& \mu_{n, 2}(x):=D_{a_{n}, b_{n}}^{[\beta]}\left((t-x)^{2} ; x\right)=\beta^{2} x^{2}+2 \frac{2 \beta^{2}-7 \beta+3}{1-\beta} \frac{b_{n}}{a_{n}} x+\left(\frac{b_{n}}{a_{n}}\right)^{2} \frac{\beta^{2}(3-\beta)}{(1-\beta)} .
\end{aligned}
$$

and

$$
\begin{aligned}
\mu_{n, 4}(x):= & D_{a_{n}, b_{n}}^{[\beta]}\left((t-x)^{4} ; x\right) \\
= & \beta^{4} x^{4}+\frac{b_{n}}{a_{n}}\left(32 \beta+22 \beta^{2}+4 \beta^{3}-34 \beta^{4}-\frac{4 \beta(2-\beta)}{1-\beta}\right) x^{3} \\
& +\left(\frac{b_{n}}{a_{n}}\right)^{2}\left(23-96 \beta-2 \beta^{2}-12 \beta^{3}+5 \beta^{4}-\frac{12 \beta^{4}}{1-\beta}+\frac{6 \beta^{2}(3-\beta)}{1-\beta}\right) x^{2} \\
& +\left(\frac{b_{n}}{a_{n}}\right)^{3}\left(42+30 \beta^{2}+30 \beta^{3}+20 \beta^{4}-\frac{10 \beta^{5}}{1-\beta}-4 \frac{\beta^{2}(3-\beta)}{(1-\beta)}\right) x+\left(\frac{b_{n}}{a_{n}}\right)^{2} \frac{\beta^{2}(3-\beta)}{(1-\beta)} .
\end{aligned}
$$

Proof of the last lemma is obvious from Lemma 1.3.

Remark 1.1. To obtain the Korovkin-type theorem we change $\beta$ with the sequence $\left(\beta_{n}\right)$ with the following property $\beta_{n} \in[0,1)$ for every $n \in \mathbb{N}$ and

$$
\lim _{n \rightarrow \infty} \beta_{n}=0 \text {. }
$$

At the rest of this paper, we will use the notation $D_{a_{n}, b_{n}}^{\left[\beta_{n}\right]}$ instead of $D_{a_{n}, b_{n}}^{[\beta]}$. Remark 1.2. Using the conditions (1.3) and (1.9) for each $0 \leq x<\infty$, we get

$$
\begin{aligned}
& \mu_{n, 1}(x)=D_{a_{n}, b_{n}}^{\left[\beta_{n}\right]}((t-x) ; x) \rightarrow 0, \quad \text { as } n \rightarrow \infty, \\
& \mu_{n, 2}(x)=D_{a_{n}, b_{n}}^{\left[\beta_{n}\right]}\left((t-x)^{2} ; x\right) \rightarrow 0, \quad \text { as } n \rightarrow \infty,
\end{aligned}
$$

and

$$
\mu_{n, 4}(x)=D_{a_{n}, b_{n}}^{\left[\beta_{n}\right]}\left((t-x)^{4} ; x\right) \rightarrow 0, \quad \text { as } n \rightarrow \infty .
$$

Lemma 1.5. For all $n \in \mathbb{N}$ and $x \in[0, \infty)$, if $\lim _{n \rightarrow \infty} \frac{a_{n}}{b_{n}} \beta_{n}=\zeta \in \mathbb{R}$, we have

$$
\begin{aligned}
& \lim _{n \rightarrow \infty} \frac{a_{n}}{b_{n}} \mu_{n, 1}(x)=-\zeta x, \\
& \lim _{n \rightarrow \infty} \frac{a_{n}}{b_{n}} \mu_{n, 2}(x)=3 x,
\end{aligned}
$$




$$
\lim _{n \rightarrow \infty}\left(\frac{a_{n}}{b_{n}}\right)^{2} \mu_{n, 4}(x)=32 \zeta x^{3}+23 x^{2}
$$

To satisfy the Korovkin-type theorem we consider the lattice homomorphism $T_{a}$ : $C[0, \infty) \rightarrow C[0, a]$ defined as $T_{a}(f)=\left.f\right|_{[0, a]}$ with a fixed $a \geq 0$. It is clear that, from Lemma 1.3 and by using the condition (1.9), we have $T_{a}\left(D_{a_{n}, b_{n}}^{\left[\beta_{n}\right]}\left(e_{k}\right)\right) \rightarrow T_{a}\left(e_{k}\right)=x^{k}$ uniformly on $[0, a]$, where $k=0,1,2$. Then, by the well-known Korovkin theorem, the following result is proven on any compact subset of $[0, \infty)$ as $n \rightarrow \infty$.

Theorem 1.1. Let $\left(D_{a_{n}, b_{n}}^{\left[\beta_{n}\right]}\right)$ be the sequence of linear positive operators given by (1.6), $f \in C[0, a]$ and $\left(\beta_{n}\right)$ be the sequence satisfying the condition (1.9). The sequence $\left(D_{a_{n}, b_{n}}^{\left[\beta_{n}\right]}\right)$ converges uniformly to $f(x)$ on $[0, a]$.

\section{Degree of Approximation}

In this section, we give an estimate the degree of approximation for the operators $D_{a_{n}, b_{n}}^{[\beta]}(f ; x)$ in terms of the modulus of continuity, Ditzian-Totik moduli of smoothness, and the Peetre's $\mathcal{K}$-functional. Also, we give Voronovskaja theorem for $D_{a_{n}, b_{n}}^{[\beta]}(f ; x)$ operators.

We begin by recalling some definitions and notations. By $C_{B}[0, \infty)$, we denote the class on real valued continuous and bounded functions $f$ defined on the interval $[0, \infty)$ with the norm $\|f\|=\sup _{x \in[0, \infty)}|f(x)|$. For $f \in C_{B}[0, \infty), \delta>0$, the $m$ th order modulus of continuity is defined as

$$
\omega_{m}(f, \delta)=\sup _{0<h \leq \delta x \in[0, \infty)} \sup _{h}\left|\Delta_{h}^{m} f(x)\right|,
$$

with $\Delta$ is the forward difference.

The Petree's $\mathcal{K}$-functional is defined by

$$
\mathcal{K}_{2}(f, \delta)=\inf _{g \in C_{B}^{2}[0, \infty)}\left\{\|f-g\|+\delta\left\|g^{\prime \prime}\right\|\right\}, \quad \delta>0,
$$

where $C_{B}^{2}[0, \infty)=\left\{g \in C_{B}[0, \infty): g^{\prime}, g^{\prime \prime} \in C_{B}[0, \infty)\right\}$ and $\|\cdot\|$ is the uniform norm on $C_{B}[0, \infty)$. By ([8], page 10$)$, we have the following inequality

$$
\mathcal{K}_{2}(f, \delta) \leq M \omega_{2}(f, \sqrt{\delta})
$$

where $M$ is a positive constant and $\omega_{2}$ is the second order modulus of smoothness for $f \in C_{B}[0, \infty)$ defined as

$$
\omega_{2}(f, \sqrt{\delta})=\sup _{0<h \leq \delta x, x+2 h \in[0, \infty)} \sup _{0}|f(x+2 h)-2 f(x+h)+f(x)| .
$$

Now, we can give the following result.

Theorem 2.1. $\left(D_{a_{n}, b_{n}}^{\left[\beta_{n}\right]}\right)$ denotes a sequence of linear positive operators defined by (1.6) and let $\left(\beta_{n}\right)$ be the sequence satisfying the condition (1.9). Then, for all $f \in C_{B}[0, \infty)$ 
and for each $x \in[0, \infty)$, the following inequality

$$
\left|D_{a_{n}, b_{n}}^{\left[\beta_{n}\right]}(f ; x)-f(x)\right| \leq 2 \omega\left(f, \delta_{a_{n}, b_{n}}^{\left[\beta_{n}\right]}\right)
$$

holds, where $\delta_{a_{n}, b_{n}}^{\left[\beta_{n}\right]}(x)=\left\{\mu_{n, 2}(x)\right\}^{1 / 2}$ and $\omega$ is usual first moduli of continuity.

Proof. For every $u, t \in[0, \infty)$ and $\delta>0$, considering the definition of modulus of continuity we can write

$$
|f(u)-f(x)| \leq\left(1+\delta^{-1}|u-x|\right) \omega(f, \delta) .
$$

Using the definition of (1.5) with the above inequality we have,

$$
\begin{aligned}
& \left|D_{a_{n}, b_{n}}^{\left[\beta_{n}\right]}(f ; x)-f(x)\right| \\
\leq & \sum_{k=1}^{\infty}\left(\int_{0}^{\infty} \theta_{\beta_{n}}\left(k-1, \frac{a_{n}}{b_{n}} u\right) d u\right)^{-1} l_{n, k}^{*}(x) \int_{0}^{\infty} \theta_{\beta_{n}}\left(k-1, \frac{a_{n}}{b_{n}} u\right)\left(1+\delta^{-1}|u-x|\right) d u \\
= & \omega(f, \delta) D_{a_{n}, b_{n}}^{\left[\beta_{n}\right]}\left(e_{0}\right)(x)+\delta^{-1} \omega(f, \delta) D_{a_{n}, b_{n}}^{\left[\beta_{n}\right]}(|u-x|)(x) .
\end{aligned}
$$

Applying Cauchy-Schwartz inequality, we have

$$
\left|D_{a_{n}, b_{n}}^{\left[\beta_{n}\right]}(f ; x)-f(x)\right| \leq \omega(f, \delta)+\delta^{-1} \omega(f, \delta)\left\{D_{a_{n}, b_{n}}^{\left[\beta_{n}\right]}\left((u-x)^{2}\right)(x)\right\}^{1 / 2} .
$$

By considering (1.8), if we choose $\delta:=\delta_{a_{n}, b_{n}}^{\left[\beta_{n}\right]}(x)$ as follows

$$
\delta_{a_{n}, b_{n}}^{\left[\beta_{n}\right]}(x)=\left\{\mu_{n, 2}(x)\right\}^{1 / 2},
$$

we obtain $(2.2)$ for each $x \in[0, \infty)$.

Now, we give the rate of convergence by means of Peetre's $\mathcal{K}$-functional.

Theorem 2.2. For each $x \in[0, \infty)$ and $f \in C_{B}[0, \infty)$, the following inequalities

$$
\begin{aligned}
\left|D_{a_{n}, b_{n}}^{\left[\beta_{n}\right]}(f ; x)-f(x)\right| & \leq 4 \mathcal{K}_{2}\left(f, d_{a_{n}, b_{n}}^{\left[\beta_{n}\right]}(x)\right)+\omega_{1}\left(f, \alpha_{a_{n}, b_{n}}^{\left[\beta_{n}\right]}\right) \\
& \leq M \omega_{2}\left(f ; \sqrt{d_{a_{n}, b_{n}}^{\left[\beta_{n}\right]}(x)}\right)+\omega_{1}\left(f, \alpha_{a_{n}, b_{n}}^{\left[\beta_{n}\right]}(x)\right)
\end{aligned}
$$

hold, where $d_{a_{n}, b_{n}}^{\left[\beta_{n}\right]}(x)=\left(\delta_{a_{n}, b_{n}}^{\left[\beta_{n}\right]}(x)\right)^{2}+\left(\alpha_{a_{n}, b_{n}}^{\left[\beta_{n}\right]}(x)\right)^{2}$ with $\delta_{a_{n}, b_{n}}^{\left[\beta_{n}\right]}(x)$ is given in Theorem 2.1, $\alpha_{a_{n}, b_{n}}^{\left[\beta_{n}\right]}(x)=\frac{b_{n}}{a_{n}} \frac{\beta_{n}\left(2-\beta_{n}\right)}{1-\beta_{n}}-\beta_{n} x$ and $M$ is a constant independently of $n$ and $x$.

Proof. Lets take auxiliary operators for $D_{a_{n}, b_{n}}^{\left[\beta_{n}\right]}$ operators as below

$$
\bar{D}_{a_{n}, b_{n}}^{\left[\beta_{n}\right]}(f ; x)=D_{a_{n}, b_{n}}^{\left[\beta_{n}\right]}(f ; x)+f(x)-f\left(\left(1-\beta_{n}\right) x+\frac{b_{n}}{a_{n}} \frac{\beta_{n}\left(2-\beta_{n}\right)}{1-\beta_{n}}\right) .
$$

So, it is obvious to see that for all $f \in C_{B}[0, \infty)$

$$
\begin{aligned}
\left|\bar{D}_{a_{n}, b_{n}}^{\left[\beta_{n}\right]}(f ; x)\right| & \leq\left|D_{a_{n}, b_{n}}^{\left[\beta_{n}\right]}(f ; x)\right|+|f(x)|+\left|f\left(\left(1-\beta_{n}\right) x+\frac{b_{n}}{a_{n}} \frac{\beta_{n}\left(2-\beta_{n}\right)}{1-\beta_{n}}\right)\right| \\
& \leq 3\|f\| .
\end{aligned}
$$


From the Lemma 1.3 we see that the new operators $\bar{D}_{a_{n}, b_{n}}^{\left[\beta_{n}\right]}(f ; x)$ obtain $\bar{D}_{a_{n}, b_{n}}^{\left[\beta_{n}\right]}(t ; x)=x$ and as a direct result of this they obtain $\bar{D}_{a_{n}, b_{n}}^{\left[\beta_{n}\right]}(t-x ; x)=0$. For $g \in C_{B}^{2}[0, \infty), t \in[0, \infty)$ by using the Taylor formula, we know

$$
g(t)=g(x)+(t-x) g^{\prime}(x)+\int_{x}^{t}(t-u) g^{\prime \prime}(u) d u .
$$

Now, we apply the operators $\bar{D}_{a_{n}, b_{n}}^{\left[\beta_{n}\right]}$ to both sides of this equality and using the equality (2.5), we get

$$
\begin{aligned}
\bar{D}_{a_{n}, b_{n}}^{\left[\beta_{n}\right]}(g ; x)-g(x)= & D_{a_{n}, b_{n}}^{\left[\beta_{n}\right]}\left(\int_{x}^{t}(t-u) g^{\prime \prime}(u) d u ; x\right) \\
& -\int_{x}^{\left(1-\beta_{n}\right) x+\frac{b_{n}}{a_{n}} \frac{\beta_{n}\left(2-\beta_{n}\right)}{1-\beta_{n}}}\left(\left(1-\beta_{n}\right) x+\frac{b_{n}}{a_{n}} \frac{\beta_{n}\left(2-\beta_{n}\right)}{1-\beta_{n}}-u\right) g^{\prime \prime}(u) d u .
\end{aligned}
$$

Now, passing absolute value and later considering the feature of norm give us following inequalities

$$
\begin{aligned}
& \left|\bar{D}_{a_{n}, b_{n}}^{\left[\beta_{n}\right]}(g ; x)-g(x)\right| \\
\leq & D_{a_{n}, b_{n}}^{\left[\beta_{n}\right]}\left(\int_{x}^{t}\left|t-u \| g^{\prime \prime}(u)\right| d u ; x\right) \\
& +\int_{x}^{\left(1-\beta_{n}\right) x+\frac{b_{n}}{a_{n}} \frac{\beta_{n}\left(2-\beta_{n}\right)}{1-\beta_{n}}}\left|\left(1-\beta_{n}\right) x+\frac{b_{n}}{a_{n}} \frac{\beta_{n}\left(2-\beta_{n}\right)}{1-\beta_{n}}-u\right|\left|g^{\prime \prime}(u)\right| d u \\
\leq & \left\|g^{\prime \prime}\right\|\left(D_{a_{n}, b_{n}}^{\left[\beta_{n}\right]}\left((t-x)^{2} ; x\right)+\left(\left(1-\beta_{n}\right) x+\frac{b_{n}}{a_{n}} \frac{\beta_{n}\left(2-\beta_{n}\right)}{1-\beta_{n}}-x\right)^{2}\right) .
\end{aligned}
$$

From Lemma 1.4, by using the (1.8) and taking $\left(-\beta_{n}\right) x+\frac{b_{n}}{a_{n}} \frac{\beta_{n}\left(2-\beta_{n}\right)}{1-\beta_{n}}:=\alpha_{a_{n}, b_{n}}^{\left[\beta_{n}\right]}(x)$, we get

$$
\begin{aligned}
D_{a_{n}, b_{n}}^{\left[\beta_{n}\right]}\left((t-x)^{2} ; x\right)+\left(\left(1-\beta_{n}\right) x+\frac{b_{n}}{a_{n}} \frac{\beta_{n}\left(2-\beta_{n}\right)}{1-\beta_{n}}-x\right)^{2} & =\left(\delta_{a_{n}, b_{n}}^{\left[\beta_{n}\right]}(x)\right)^{2}+\left(\alpha_{a_{n}, b_{n}}^{\left[\beta_{n}\right]}(x)\right)^{2} \\
& =d_{a_{n}, b_{n}}^{\left[\beta_{n}\right]}(x) .
\end{aligned}
$$

Using the inequality (2.7) and equality (2.8) we can write

$$
\left|\bar{D}_{a_{n}, b_{n}}^{\left[\beta_{n}\right]}(g ; x)-g(x)\right| \leq\left(d_{a_{n}, b_{n}}^{\left[\beta_{n}\right]}(x)\right)\left\|g^{\prime \prime}\right\| .
$$

For $f \in C_{B}[0, \infty)$ and considering (1.5), we can write

$$
\left|D_{a_{n}, b_{n}}^{\left[\beta_{n}\right]}(f ; x)\right|
$$




$$
\begin{aligned}
& \leq \sum_{k=1}^{\infty}\left(\int_{0}^{\infty} \theta_{\beta}\left(k-1, \frac{a_{n}}{b_{n}} u\right) d u\right)^{-1} l_{n, k}^{*}(x) \int_{0}^{\infty} \theta_{\beta}\left(k-1, \frac{a_{n}}{b_{n}} u\right)|f(u)| d u+2^{-\frac{a_{n}}{b_{n}} x}|f(0)| \\
& \leq\|f\| \sum_{k=1}^{\infty}\left(\int_{0}^{\infty} \theta_{\beta}\left(k-1, \frac{a_{n}}{b_{n}} u\right) d u\right)^{-1} l_{n, k}^{*}(x) \int_{0}^{\infty} \theta_{\beta}\left(k-1, \frac{a_{n}}{b_{n}} u\right) d u+2^{-\frac{a_{n}}{b_{n}} x} \\
(2.10) \quad & =\|f\| D_{a_{n}, b_{n}}^{\left[\beta_{n}\right]}\left(e_{0} ; x\right)=\|f\| .
\end{aligned}
$$

Combining (2.9) and (2.6), for $f \in C_{B}[0, \infty)$ and for $g \in C_{B}^{(2)}[0, \infty)$, we have

$$
\begin{aligned}
\left|D_{a_{n}, b_{n}}^{\left[\beta_{n}\right]}(f ; x)-f(x)\right| \leq & \left|\bar{D}_{a_{n}, b_{n}}^{\left[\beta_{n}\right]}((f-g) ; x)\right|+|(f-g)(x)|+\left|\bar{D}_{a_{n}, b_{n}}^{\left[\beta_{n}\right]}(g ; x)-g(x)\right| \\
& +\left|f\left(\left(1-\beta_{n}\right) x+\frac{b_{n}}{a_{n}} \frac{\beta_{n}\left(2-\beta_{n}\right)}{1-\beta_{n}}\right)-f(x)\right| \\
& \leq 4\|f-g\|+\left(d_{a_{n}, b_{n}}^{\left[\beta_{n}\right]}(x)\right)\left\|g^{\prime \prime}\right\|+\left|f\left(\left(1-\beta_{n}\right) x+\frac{b_{n}}{a_{n}} \frac{\beta_{n}\left(2-\beta_{n}\right)}{1-\beta_{n}}\right)-f(x)\right| .
\end{aligned}
$$

Taking the infimum over all $g \in C_{B}^{2}[0, \infty)$, we reach the result $(2.4)$ and by using the inequality (2.1) we find, for each $x \in[0, \infty)$

$$
\left|D_{a_{n}, b_{n}}^{\left[\beta_{n}\right]}(f ; x)-f(x)\right| \leq 4 M \omega_{2}\left(f, d_{a_{n}, b_{n}}^{\left[\beta_{n}\right]}(x)\right)+\omega_{1}\left(f, \alpha_{a_{n}, b_{n}}^{\left[\beta_{n}\right]}(x)\right),
$$

which implies the proof.

Now we give the result by using Ditzian-Totik moduli of smoothness. Let start with reminding the some definitions which will be used.

Let function $f \in C[0, \infty)$ and if we take step weight function $\phi:[0, \infty) \rightarrow \mathbb{R}$. The first order Ditzian-Totik modulus of smoothness and corresponding $K$-functional are given by, respectively,

$$
\begin{aligned}
& \omega_{1}^{\phi}(f, \sqrt{\delta})=\sup _{0<h \leq \sqrt{\delta}}\left\{\left|f\left(x+\frac{h \phi(x)}{2}\right)+f\left(x-\frac{h \phi(x)}{2}\right)\right|: x \pm \frac{h \phi(x)}{2} \in[0, \infty)\right\}, \\
& \mathcal{K}_{1, \phi}(f, \delta)=\inf \left\{\|f-g\|_{\infty}+\delta\left\|\phi g^{\prime}\right\|_{\infty}: g \in C^{\prime}(\phi)\right\}, \quad \delta>0,
\end{aligned}
$$

where $C(\phi)=\left\{g \in A C_{l o c}[0, \infty):\left\|\phi g^{\prime}\right\|_{\infty}<\infty\right\} . g \in A C_{l o c}[0, \infty)$ shows that the function $g$ is differentiable and $g$ is absolutely continuous on every closed interval $[a, b] \subset[0, \infty)$. It is known that there exists a positive constant $M>0$, such that (see [8], p.68)

$$
\frac{1}{M} \omega_{1}^{\phi}(f, \sqrt{\delta}) \leq \mathcal{K}_{1, \phi}(f, \delta) \leq M \omega_{1}^{\phi}(f, \sqrt{\delta})
$$

Theorem 2.3. Let $f \in C_{B}[0, \infty)$. For $x \in(0, \infty)$, we have

$$
\left\|D_{a_{n}, b_{n}}^{\left[\beta_{n}\right]}(f)-f\right\| \leq 2 \mathcal{K}_{1, \phi}\left(f, \delta_{a_{n}, b_{n}}^{\left[\beta_{n}\right]}\right) \leq 2 M \omega_{1}^{\phi}\left(f, \sqrt{\delta_{a_{n}, b_{n}}^{\left[\beta_{n}\right]}}\right),
$$

where $\phi(x)=\sqrt{x}$ is a step function. 
Proof. For $g \in C(\phi)$, by using Taylor's formula of $g$, we have

$$
g(t)=g(x)+\int_{x}^{t} g^{\prime}(u) d u=g(x)+\int_{x}^{t} \frac{g^{\prime}(u)}{\phi(u)} \phi(u) d u .
$$

Then, for the step function $\phi(x)=\sqrt{x}$, we get

$$
|g(t)-g(x)| \leq\left\|\phi g^{\prime}\right\|_{\infty}\left|\int_{x}^{t} \frac{1}{\phi(u)} d u\right|=\left\|\phi g^{\prime}\right\|_{\infty} 2|\sqrt{t}-\sqrt{x}|=2\left\|\phi g^{\prime}\right\|_{\infty} \frac{|t-x|}{\sqrt{t}+\sqrt{x}} .
$$

From the inequality $\sqrt{t}+\sqrt{x} \geq \sqrt{x}$, we get

$$
|g(t)-g(x)| \leq 2\left\|\phi g^{\prime}\right\|_{\infty} \frac{|t-x|}{\sqrt{x}}=2\left\|\phi g^{\prime}\right\|_{\infty} \frac{|t-x|}{\phi(x)} .
$$

Using (2.13) and (2.10), for $f \in C_{B}[0, \infty)$ and $g \in C(\phi)$, we have

$$
\begin{aligned}
\left|D_{a_{n}, b_{n}}^{\left[\beta_{n}\right]}(f ; x)-f(x)\right| & \leq\left|D_{a_{n}, b_{n}}^{\left[\beta_{n}\right]}((f-g) ; x)\right|+\left|D_{a_{n}, b_{n}}^{\left[\beta_{n}\right]}(g ; x)-g(x)\right|+|g(x)-f(x)| \\
& \leq 2\|f-g\|+2 \frac{\left\|\phi g^{\prime}\right\|_{\infty}}{\phi(x)} D_{a_{n}, b_{n}}^{\left[\beta_{n}\right]}(|t-x| ; x) .
\end{aligned}
$$

By applying Cauchy-Schwartz inequality, we can write

$$
\begin{aligned}
\left|D_{a_{n}, b_{n}}^{\left[\beta_{n}\right]}(f ; x)-f(x)\right| & \leq 2\|f-g\|+2 \frac{\left\|\phi g^{\prime}\right\|_{\infty}}{\phi(x)}\left(D_{a_{n}, b_{n}}^{\left[\beta_{n}\right]}\left((t-x)^{2} ; x\right)\right)^{1 / 2} \\
& \leq 2\|f-g\|+2 \frac{\left\|\phi g^{\prime}\right\|_{\infty}}{\phi(x)} \delta_{a_{n}, b_{n}}^{\left[\beta_{n}\right]}(x) .
\end{aligned}
$$

Taking the infimum on the right hand side over all $g \in C^{2}(\phi)$ we obtain

$$
\left|D_{a_{n}, b_{n}}(f ; x)-f(x)\right| \leq 2 \mathcal{K}_{1, \phi}\left(f, \delta_{a_{n}, b_{n}}^{\left[\beta_{n}\right]}\right) .
$$

Considering (2.11) we get (2.12) which is desired result.

In this section, we obtain some pointwise estimates of rate of convergence of the operators (1.6). The Lipschitz-type space is given as follow, in [13];

$$
\operatorname{Lip}_{M}^{*}(\eta)=\left\{f \in C[0, \infty):|f(t)-f(x)| \leq M_{f} \frac{|t-x|^{\eta}}{(t+x)^{\frac{\eta}{2}}}, x, t \in(0, \infty)\right\},
$$

where $M_{f}$ is a positive constant and $\eta \in(0,1]$.

Theorem 2.4. Let $f \in \operatorname{Lip}_{M}^{*}(\eta)$. Then, for all $x \in(0, \infty)$, we get

$$
\left|D_{a_{n}, b_{n}}^{\left[\beta_{n}\right]}(f ; x)-f(x)\right| \leq M\left(\frac{\mu_{n, 2}(x)}{x}\right)^{\eta / 2}
$$

where $\mu_{n, 2}(x)$ is the same as in Lemma 1.4 . 
Proof. For a function $f \in \operatorname{Lip}_{M}^{*}(\eta)$, by using the definition, we get

$$
|f(t)-f(x)| \leq M_{f} \frac{|t-x|^{\eta}}{(t+x)^{\frac{\eta}{2}}} .
$$

Applying the operators $D_{a_{n}, b_{n}}^{\left[\beta_{n}\right]}$ on both sides of the above inequality, we have

$$
\left|D_{a_{n}, b_{n}}^{\left[\beta_{n}\right]}(f ; x)-f(x)\right| \leq M_{f} D_{a_{n}, b_{n}}^{\left[\beta_{n}\right]}\left(\frac{|t-x|^{\eta}}{(t+x)^{\frac{\eta}{2}}} ; x\right) .
$$

By using the Hölder's inequality, with $p=\frac{2}{\eta}, q=\frac{2}{2-\eta}$ and, using the Lemma 1.4, we can write

$$
\begin{aligned}
\left|D_{a_{n}, b_{n}}^{\left[\beta_{n}\right]}(f ; x)-f(x)\right| & \leq M_{f}\left(D_{a_{n}, b_{n}}^{\left[\beta_{n}\right]}\left(\frac{(t-x)^{2}}{(t+x)} ; x\right)\right)^{\frac{\eta}{2}}\left(D_{a_{n}, b_{n}}^{\left[\beta_{n}\right]}(1 ; x)\right)^{\frac{2-\eta}{2}} \\
& \leq M_{f} \frac{1}{x^{\eta / 2}}\left(D_{a_{n}, b_{n}}^{\left[\beta_{n}\right]}\left((t-x)^{2} ; x\right)\right)^{\frac{\eta}{2}}
\end{aligned}
$$

So, we obtain (2.14).

Now we give an estimate for the rate of convergence by the means of the general space of the Lipschitz-type maximal functions. Let $\widetilde{C}_{B}[0, \infty)$ be the space of bounded, uniformly continuous real valued functions on $[0, \infty)$. The Lipschitz-type maximal function of order $\eta$ of $f \in \widetilde{C}_{B}[0, \infty)$ is introduced by Lenze [11] as

$$
\widetilde{f_{\eta}}(x)=\sup _{t \neq x, t \in[0, \infty)} \frac{|f(t)-f(x)|}{|t-x|^{\eta}}, \quad x \in[0, \infty),
$$

and $\eta \in(0,1]$.

Theorem 2.5. Let $\left(D_{a_{n}, b_{n}}^{\left[\beta_{n}\right]}\right)$ be a sequence of linear positive operators defined by (1.6). Then, for all $f \in \widetilde{C}_{B}[0, \infty)$, we get

$$
\left|D_{a_{n}, b_{n}}^{\left[\beta_{n}\right]}(f ; x)-f(x)\right| \leq \widetilde{f_{\eta}}(x)\left(\delta_{a_{n}, b_{n}}^{\left[\beta_{n}\right]}(x)\right)^{\eta},
$$

where $\delta_{a_{n}, b_{n}}^{\left[\beta_{n}\right]}$ is the same as in Theorem 2.1 .

Proof. Using the definition of maximal function, we get

$$
|f(t)-f(x)| \leq \widetilde{f_{\eta}}(x)|t-x|^{\eta}
$$

and applying the operators $D_{a_{n}, b_{n}}^{\left[\beta_{n}\right]}$ on both sides of this equation, we get

$$
\left|D_{a_{n}, b_{n}}^{\left[\beta_{n}\right]}(f ; x)-f(x)\right| \leq \widetilde{f_{\eta}}(x) D_{a_{n}, b_{n}}^{\left[\beta_{n}\right]}\left(|t-x|^{\eta} ; x\right) .
$$

Applying the Hölder's inequality with $p=\frac{2}{\eta}$ and $q=\frac{2}{2-\eta}$, using Lemma 1.3, it follows that

$$
\begin{aligned}
D_{a_{n}, b_{n}}^{\left[\beta_{n}\right]}\left(|t-x|^{\eta} ; x\right) & \leq \widetilde{f_{\eta}}(x)\left(D_{a_{n}, b_{n}}^{\left[\beta_{n}\right]}\left(|t-x|^{2} ; x\right)\right)^{\frac{\eta}{2}}\left(D_{a_{n}, b_{n}}^{\left[\beta_{n}\right]}\left(1^{\frac{2-\eta}{2}} ; x\right)\right)^{\frac{2-\eta}{2}} \\
& \leq \widetilde{f_{\eta}}(x)\left(D_{a_{n}, b_{n}}^{\left[\beta_{n}\right]}\left((t-x)^{2} ; x\right)\right)^{\frac{\eta}{2}}
\end{aligned}
$$




$$
\leq \widetilde{f_{\eta}}(x)\left(\delta_{a_{n}, b_{n}}^{\left[\beta_{n}\right]}(x)\right)^{\eta} .
$$

Hence, the proof is completed.

\section{Voronovskaja Theorem}

We prove a Voronoskaja type theorem for the operators $D_{a_{n}, b_{n}}^{\left[\beta_{n}\right]}(f ; x)$.

Theorem 3.1. For every $f \in C_{B}[0, \infty)$ such that $f^{\prime}, f^{\prime \prime} \in C_{B}[0, \infty)$, and for every fixed $x \in[0, \infty)$, we have

$$
\lim _{n \rightarrow \infty} \frac{a_{n}}{b_{n}}\left(D_{a_{n}, b_{n}}^{\left[\beta_{n}\right]}(f ; x)-f(x)\right)=-\zeta x f^{\prime}(x)+\frac{3}{2} x f^{\prime \prime}(x),
$$

where $\zeta$ is the same with the Lemma 1.5.

Proof. Let $x \in[0, \infty)$ be a fixed point. For all $t \in[0, \infty)$, by using Taylor expansion we have

$$
f(t)=f(x)+(t-x) f^{\prime}(x)+\frac{1}{2}(t-x)^{2} f^{\prime \prime}(x)+R(t, x)(t-x)^{2},
$$

where $R(t, x)$ is the remainder term, $R(t, x) \in C_{B}[0, \infty)$, and $R(t, x) \rightarrow 0$ as $t \rightarrow x$. Applying the operator $D_{a_{n}, b_{n}}^{\left[\beta_{n}\right]}$ to both sides of Taylor expansion and considering $D_{a_{n}, b_{n}}^{\left[\beta_{n}\right]}\left(e_{0} ; x\right)=$ 1 , we get

$$
\begin{aligned}
\frac{a_{n}}{b_{n}}\left(D_{a_{n}, b_{n}}^{\left[\beta_{n}\right]}(f ; x)-f(x)\right)= & \frac{a_{n}}{b_{n}} D_{a_{n}, b_{n}}^{\left[\beta_{n}\right]}((t-x) ; x) f^{\prime}(x)+\frac{1}{2} \frac{a_{n}}{b_{n}} D_{a_{n}, b_{n}}^{\left[\beta_{n}\right]}\left((t-x)^{2} ; x\right) f^{\prime \prime}(x) \\
& +\frac{a_{n}}{b_{n}} D_{a_{n}, b_{n}}^{\left[\beta_{n}\right]}\left(R(t, x)(t-x)^{2} ; x\right) \\
= & A_{1}+A_{2}+A_{3} .
\end{aligned}
$$

Thus, we immediately have

$$
\begin{aligned}
& A_{1}=\frac{a_{n}}{b_{n}} \mu_{n, 1}(x) f^{\prime}(x), \\
& A_{2}=\frac{1}{2} \frac{a_{n}}{b_{n}} \mu_{n, 2}(x) f^{\prime \prime}(x) .
\end{aligned}
$$

Now, we estimate $A_{3}$. From Cauchy-Schwartz inequality, we have

$$
\begin{aligned}
A_{3} & =\frac{a_{n}}{b_{n}} D_{a_{n}, b_{n}}^{\left[\beta_{n}\right]}\left(R(t, x)(t-x)^{2} ; x\right) \\
& \leq\left\{D_{a_{n}, b_{n}}^{\left[\beta_{n}\right]}\left((R(t, x))^{2} ; x\right)\right\}^{1 / 2}\left\{\left(\frac{a_{n}}{b_{n}}\right)^{2} D_{a_{n}, b_{n}}^{\left[\beta_{n}\right]}\left((t-x)^{4} ; x\right)\right\}^{1 / 2} .
\end{aligned}
$$

The properties of the function $R(t, x)$ implies that $R^{2}(x, x)=0$ and $R^{2}(x, x) \in C_{B}[0, \infty)$. Hence, we obtain

$$
\lim _{n \rightarrow \infty} D_{a_{n}, b_{n}}^{\left[\beta_{n}\right]}\left((R(t, x))^{2} ; x\right)=R^{2}(x, x)=0, \quad x \in[0, \infty) .
$$

Furthermore, by applying equation (1.12) from Lemma 1.5, we get

$$
\lim _{n \rightarrow \infty} D_{a_{n}, b_{n}}^{\left[\beta_{n}\right]}\left(R(t, x)(t-x)^{2} ; x\right)=0 .
$$


Moreover, if we take limits as $n \rightarrow \infty$ over $A_{1}$ and $A_{2}$, from the equalities (1.10) and (1.11), this implies the desired result.

\section{REFERENCES}

[1] O. Agratini, On a sequence of linear and positive operators, Facta Univ. Ser. Math. Inform. 14 (1999), 41-48.

[2] O. Agratini, On the rate of convergence of a positive approximation process, Nihonkai Math. J. 11 (2000), 47-56.

[3] A. Erençin and F. Taşdelen, On a family of linear and positive operators in weighted spaces, Journal of Inequalities in Pure and Applied Mathematics 8(6) (2007), 2-39.

[4] A. Erençin and F. Taşdelen, On certain Kantorovich type operators, Fasc. Math. 41 (2009), 65-71.

[5] N. K. Govil, V. Gupta, and D. Soybaş, Certain new classes of Durrmeyer type operators, Appl. Math. Comput. 225 (2013), 195-203.

[6] V. Gupta and G. C. Greubel, Moment Estimations of new Szász-Mirakyan-Durrmeyer operators, Appl. Math. Comput. 271 (2015), 540-547.

[7] V. Gupta and G.Tachev, Approximation with Positive Linear Operators and Linear Combinations, Springer, Cham, 2017.

[8] V. Gupta and R. P. Agarwal, Convergence Estimates in Approximation Theory, Springer, USA, New York, 2014.

[9] N. Ispir and N. Manav, Approximation by the summation integral type operators based on Lupaş-Szász basis functions, Journal of Science and Art 4(45), 2018, 853-868.

[10] G. C. Jain, Approximation of functions by a new class of linear operators, J. Aust. Math. Soc. 13(3) (1972), 271-276.

[11] B. Lenze, Bernstein-Baskakov-Kantorovich operators and Lipschitz-type maximal functions, Colloq. Math. Soc. János Bolyai 58 (1990), 469-496.

[12] A. Lupaş, The approximation by some positive linear operators, in: M. W. Müller et al., (Eds.), Proceedings of the International Dortmund Meeting on Approximation Theory, Akademie Verlag, Berlin, 1995, 201-229.

[13] M. A. Özarslan and O. Duman, Local approximation behavior of modified SMK operators, Miskolc Math. Notes 11(1) (2010), 87-89.

${ }^{1}$ Department of Mathematics,

GAZI UNIVERSITY,

06500 Teknikokullar, Ankara, Turkey

Email address: nmanav@gazi.edu.tr

Email address: nispir@gazi.edu.tr 